\title{
OSCILLATING STAR FORMATION
}

\author{
G. BODIFEE \\ Astrophysica1 Institute \\ Vrije Universiteit Brussel \\ Brussels, Belgium.
}

\section{ABSTRACT .}

As a consequence of positive feedback effects in interstellar chemical and star forming processes, a star formation region may undergo nonlinear oscillations.

A model has been built of a star formation region in which mass transformation processes take place, regulated by a throughflow of matter and the interactions between the components of the system. In this approach, a star formation region is regarded as a galactic dissipative structure (Nicolis and Prigogine,1977), sustaining itself as a more or less stable, ordered, non-equilibrium system, independent of the of the environment, except for supply of material and the removal of waste. The system of the model includes three components:

Cool atomic gas. This gas does not lead to collapse; no star formation is possible.

Cool and dusty molecular clouds. Due to efficient molecular cooling, this gas can collapse. However, external pressure by expanding HII regions applied for a collapse to set in.

Young stars with their HII regions. The stars are able to trigger further star formation in molecular clouds (Elmegreen \& Lada, 1977).

It is assumed that the total mass of the system is constant:

The system is attached to two "reservoirs":

- cool atomic gas is available to replenish the mass that is removed from the system.

- matter that is permanently buried in stellar remnants as low-mass main sequence stars, is removed from the system ("waste" reservoir). Mass transformations between components are described by a systein of parameterized equations.

$$
\frac{d X}{d t}=F(X)
$$

where $X$ is a vector of the component concentrations, and $F$ a vector of nonlinear polyonomial and autonomous equations, that describe the transformation processes. 
THE PROCESS SYSTEM.

1. Inflow of atomic gas. This compensates for mass that leaves the system as old stars

$$
\frac{\mathrm{d}_{1} \mathrm{~A}}{\mathrm{dt}}=-\frac{\mathrm{d}_{2} \mathrm{~S}}{\mathrm{dt}}=\mathrm{K}_{1} \mathrm{~S}
$$

2. Stellar evolution

$$
\frac{\mathrm{d}_{2} \mathrm{R}}{\mathrm{dt}}=-\frac{\mathrm{d}_{2} \mathrm{~S}}{\mathrm{dt}}=\mathrm{K}_{1} \mathrm{~S}
$$

3. Mass loss of young stars and recombination of ionized gas

$$
\frac{\mathrm{d}_{3} \mathrm{~A}}{\mathrm{dt}}=-\frac{\mathrm{d}_{3} \mathrm{~S}}{\mathrm{dt}}=\mathrm{K}_{2} \mathrm{~S}
$$

4. Spontaneous star formation. Low-mass stars, that do not trigger further star formation, are themselves formed spontaneously.

$$
\frac{\mathrm{d}_{4} \mathrm{R}}{\mathrm{dt}}=-\frac{\mathrm{d}_{4} \mathrm{M}}{\mathrm{dt}}=\mathrm{K}_{3} \mathrm{M}^{\mathrm{n}_{1}}
$$

5. Production of interstellar molecular gas. Molecular cooling allows cloud to collapse, leading to larger densities and faster reaction rates.

$$
\frac{d_{5} M}{d t}=-\frac{d_{5} A}{d t}=K_{4} A^{n_{2}}(M+A){ }^{n_{3}} s^{-n_{4}}
$$

6. Triggered star formation.

$$
\frac{\mathrm{d}_{6} \mathrm{~S}}{\mathrm{dt}}=-\frac{\mathrm{d}_{6} \mathrm{M}}{\mathrm{dt}}=\mathrm{K}_{5} \mathrm{SM}^{\mathrm{n}_{1}}
$$

Different types of system behaviour occur, depending on the parameter values. Within certain ranges the stationary state is stable, and the systen evolves towards this state in a direct or oscillating way. Beyond some critical parameter values, the stationary state is unstable and self-sustained oscillations develop that behave like a limit cycle.

RESULTS OF CALCULATIONS.

Limit cycle oscillations occur for small values of the exponents $n_{1}$ and $\mathrm{n}_{2}$ (both smaller than 2), and large values of $\mathrm{n}_{3}$. The influence of the stellar radiation on molecular production, as expressed by $n_{4}$, has no effect on the oscillations. Atomic cooling dampens any oscillation drastically. For $\geq 0.2$, no limit cycle can occur, whatever the values of the other parameters. The efficiency coefficient for triggered star formation has a strong influence: for small values the system evolves towards a stationary state after some initial oscillations. For increasing triggered star formation efficiency, the amplitude of the oscillations increase until eventually a limit cycle is established.

\section{REFERENCES .}

Elmegreen, B.G., Lada, C.J., 1977, Astrophys.J. 214, 725.

Nicolis, G., Prigogine, I., 1977, Self-organisation in Nonequilibrium Systems, Wiley Intersciences, New York. 